\title{
Interrelation of Chemistry and Process Design in Biodiesel Manufacturing by Heterogeneous Catalysis
}

\author{
Alexandre C. Dimian - Zbig W. Srokol • \\ Marjo C. Mittelmeijer-Hazeleger • Gadi Rothenberg
}

Published online: 28 May 2010

(c) The Author(s) 2010. This article is published with open access at Springerlink.com

\begin{abstract}
The pros and cons of using heterogeneous catalysis for biodiesel manufacturing are introduced, and explained from a chemistry and engineering viewpoint. Transesterification reactions of various feed types are then compared in batch and continuous process operation modes. The results show that the reaction chemistry and process kinetics characterising a particular feedstock are determinant factors for obtaining high-grade biodiesel. When using heterogeneous catalysis, the biodiesel quality of a particular feed can be controlled by customising the process design and operation conditions.
\end{abstract}

Keywords Biodiesel - Heterogeneous catalysis ·

Process design - Reactor technology $\cdot$ Chemical kinetics

\section{Introduction}

The ongoing debate of oil for food or fuel notwithstanding, today's biodiesel technology leaves much to be desired. It is dominated by batch processes using homogeneous acid/ base catalysis. The batch mode offers simple scale-up of laboratory recipes. It also ensures good flexibility of operation faced to a large chemical variability of raw materials. However, these process also suffer from three serious disadvantages: low productivity, high energy costs,

A. C. Dimian $(\bowtie) \cdot$ Z. W. Srokol

Yellowdiesel B.V., P.O. Box 2535, 1000 CM Amsterdam,

The Netherlands

e-mail: a.c.dimian@yellowdiesel.com

M. C. Mittelmeijer-Hazeleger · G. Rothenberg

Van 't Hoff Institute for Molecular Sciences, University

of Amsterdam, Nieuwe Achtergracht 166, 1018 WV Amsterdam,

The Netherlands and large amounts of aqueous waste. In fact, the cost of product separation, waste disposal and catalyst neutralisation is the main factor that renders current biodiesel production uneconomical.

Interestingly, there is a simple and elegant solution to these problems: Continuous biodiesel manufacturing using heterogeneous catalysis. Esterification and transesterification reactions proceed readily over solid acid/base catalysts [1]. Importantly, such processes have practically no aqueous waste streams, and require no catalyst neutralising. Moreover, they incur lower capital expenses (CapEx) and lower operational expenses (OpEx). Thus, compared to a batch homogeneous process, a continuous process using a solid catalyst can save $40-50 \%$ in capital costs and $30-60 \%$ in energy.

Despite these advantages, biodiesel manufacturing using heterogeneous catalysts remains an emerging technology [2], with only one major licensor [3]. Previously, our group demonstrated how reactive distillation technology can be employed to produce biodiesel from feedstock containing large amounts of free fatty acids (up to $100 \%$ FFA), using a super-acid heterogeneous catalyst [4-6]. However, for a conventional low-FFA triglyceride feedstock, such as vegetal oils, a base heterogeneous catalyst is more appropriate. This is because one has to mainly catalyse transesterification, rather than esterification. The task is relatively simple on a lab scale, when starting from clean (analytical grade) oils. The real challenge of the process design is dealing with industrial (technical grade) chemically varied feedstock that must be converted into a product with strict quality specifications, as defined by EN14212 and ASTM D6751.

The overall transesterification reaction involves three equilibrium reaction steps in which intermediate species are formed (Scheme 1). In these steps, the resulting di- and mono-glycerides content is fixed. It cannot be corrected 


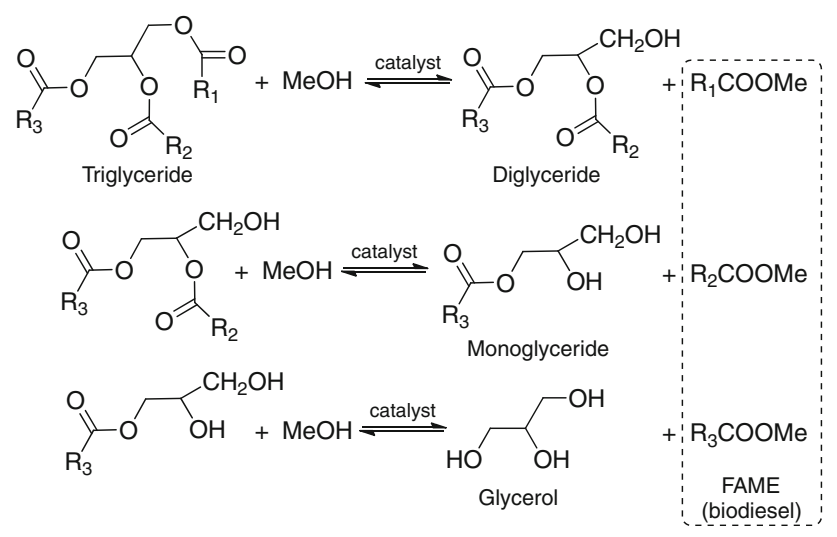

Scheme 1 The three equilibrium reactions that comprise the transesterification of glycerides

afterwards by any economical separation technique. This is important, as the key biodiesel specifications (in wt $\%$ ) are: ester content min. 96.5\%, mono-glycerides max. $0.8 \%$, di-glycerides $\max 0.2 \%$, triglyceride max. $0.2 \%$, free glycerine max. $0.02 \%$, and total glycerine $\max 0.25 \%$. Another restriction is a ceiling of max $12 \%$ linolenic acid methylester. The residual triglyceride and glycerol levels are determined in both reaction and separation steps, but again related to the feedstock composition and catalyst activity. This means that a good biodiesel manufacturing process should achieve a suitable mixture composition after each reaction stage.

Responding to this challenge, we now developed a reactor technology based on variable residence time, that operating at higher pressure/higher temperature [7]. A continuous operation microplant was built for getting fundamental data for process design. Here we describe the relation between the feed composition, the operating parameters of the reactor, and the catalyst performance, in terms of activity, species distribution, long-term robustness and regeneration.

\section{Results and Discussion}

\subsection{Stoichiometry and Reaction Mechanism}

The overall transesterification consists of three equilibrium reaction steps (Scheme 1). This means that the minimum stoichiometric alcohol:triglyceride ratio is $3: 1$. In practice, the ratio is often set higher, facilitating substrate conversion and improving flow properties. Here we kept it constant at 9:1. The reaction mechanism follows a LangmuirHinshelwood route: physisorption, chemisorption, surface reaction, and product desorption [8]. Initially, an alkoxide anion forms by the chemisorption of methanol on the catalyst active site. Then the first tetrahedral intermediate

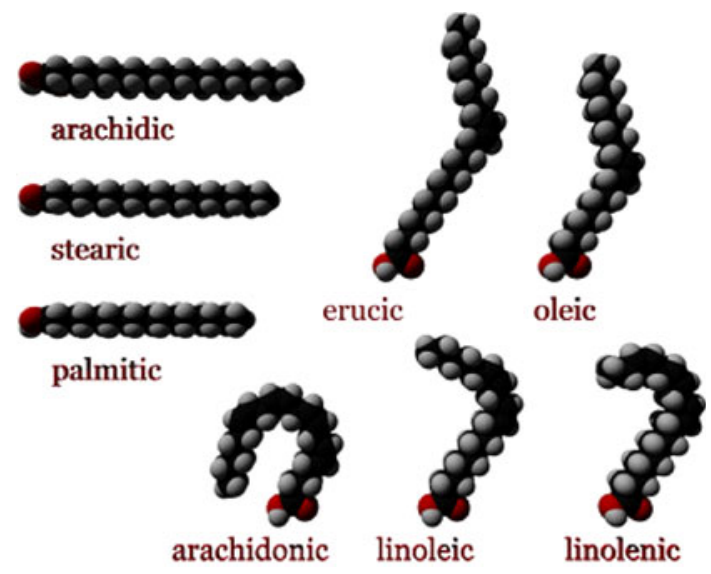

Fig. 1 Spatial conformations of various fatty acid hydrocarbon chains

forms via nucleophilic attack on the glyceride carbonyl group. Release from the active site generates a second tetrahedral intermediate, from which the fatty ester and glycerol substitute result by molecular rearrangement.

The structural conformation of the hydrocarbon chain plays an important role in the kinetics. Saturated chains are straight, allowing the molecules to slip easily through the large catalyst pores. The conformations of unsaturated chains are more complicated (see Fig. 1). The ' $\Omega-9$ oleic and erucic acids shows a V-shape, whereas the polyunsaturated linoleic and linolenic acids are "hook"-shaped. In-pore diffusion is made difficult by these bends. Moreover, attraction forces between the pi orbitals of the double bonds and the catalyst surface hinder the diffusion of the unsaturated molecules.

\subsection{Surface Porosity}

Our solid base catalyst is a mixed metal oxide. Its internal structure was characterised using a Thermo Fischer Scientific mercury porosimeter. The key results are: bulk density $1.04 \mathrm{~g} / \mathrm{cm}^{3}$, void volume $680 \mathrm{~mm}^{3} / \mathrm{g}$, accessible porosity $58 \%$, internal area $90 \mathrm{~m}^{2} / \mathrm{g}$, average pore diameter $25 \mathrm{~nm}$, median pore diameter $63 \mathrm{~nm}$, and maximum pore diameter $275 \mathrm{~nm}$. The size distribution shows rather large pores, favouring the diffusion of the bulky glyceride molecules (Fig. 2).

\subsection{Comparing Transesterification of Different Feedstocks}

Table 1 gives the composition of the four oils studied: rapeseed oil, commercial frying fat, soya oil, and peanut oil. Importantly, all technical grade oils were pretreated prior to reaction, thus avoiding any impurities that may affect the reaction kinetics. The chemical characteristic of 


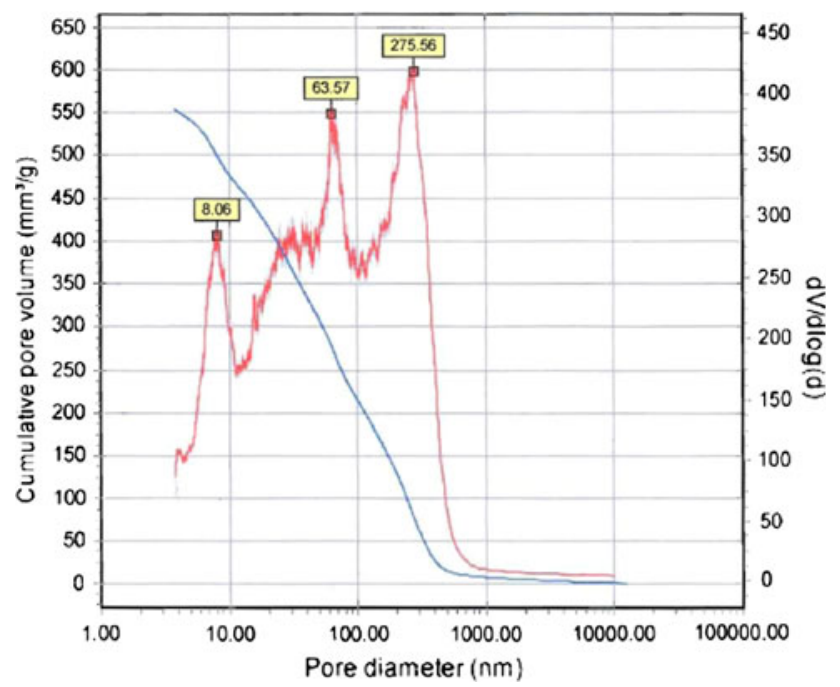

Fig. 2 Pore size distribution of the solid base mixed oxide catalyst

oils are related to the ratio of unsaturated to saturated fatty acids, $R$. This ratio varies by a whole order of magnitude from frying fat to rapeseed oil.

Figure 3 presents typical concentration profiles of ester over time, obtained under batch conditions using a solid base catalyst (see Sect. 4 for details). The products are grouped in two categories, namely saturated $\mathrm{C}_{16}-\mathrm{C}_{18}$ and unsaturated $\mathrm{C}_{18+}$, for clarity. The temperature reaches a steady-state after about $45 \mathrm{~min}$. All the reactions showed first a sluggish initial period of about $30 \mathrm{~min}$. This may be a temperature stabilising effect in the autoclave, and/or may be caused by the high viscosity of the triglyceride feed that hinders in-pore diffusion. The viscosity diminishes at higher temperatures and by mixing with freshly formed ester. After $60 \mathrm{~min}$, the slope of the curve gives a good indication of the continuous steady-state reaction rate. The reaction reaches equilibrium after 120-150 min, depending on the oil composition.

Figure 4 compares the kinetic behaviour of the four oils. The slope of the curves shows that the frying fat exhibits the fastest reaction, followed by rapeseed oil, soybean and peanut oils, the last one with a somewhat longer sluggish period. Considering only the linear range of the isothermal data gives a relative reaction rate of fat/rapeseed/soy/peanut as 1.6/1.26/1.17/1.0. These results suggest that the

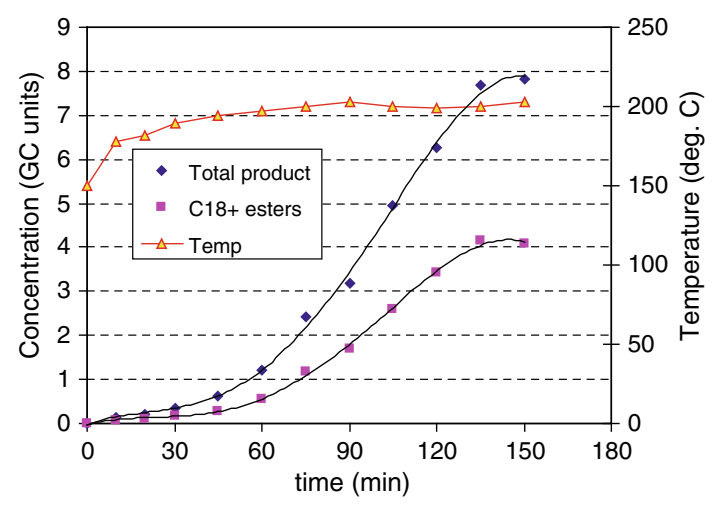

Fig. 3 Transesterification profiles of rapeseed oil to fatty acid methyl esters (FAME) and glycerol in a batch autoclave reactor (see Sect. 4 for details)

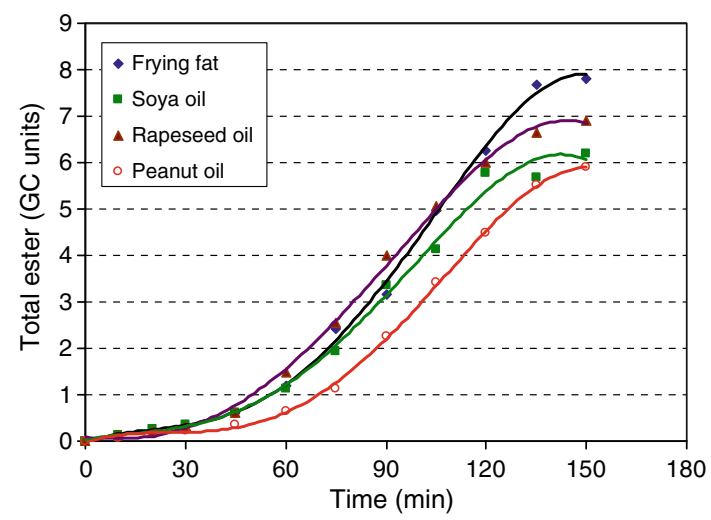

Fig. 4 Comparing the transesterification of four raw materials

chemical nature of the feedstock should influence the kinetics of the transesterification reaction by heterogeneous catalysis. Faster reaction rates should be expected by higher content in saturated fatty acids, while the opposite should happen by highly unsaturated oils. Indeed, the behaviour of frying fat (lowest $R, 0.75$ ) confirms this hypothesis, but that of the rapeseed oil $(R=9)$ does not. Note, however, that rapeseed oil has the highest content of $\mathrm{C}_{18}: 1$ oleic acid. Soy and peanut oils $(R \sim 9)$, exhibit the slowest kinetics. Soy oil has a higher content of $\mathrm{C}_{18}: 2$ linoleic acid, while peanut oil contains some longer hydrocarbon chains (saturated and unsaturated), grouped as $\mathrm{C}_{20+}$.
Table 1 Fatty acid composition of feedstock

a The fatty acid notation $\mathrm{C}_{\mathrm{n}}: \mathrm{m}$ gives the chain length $n$ and the degree of unsaturation (number of double bonds), $m$

\begin{tabular}{llllllll}
\hline Feedstock & \multicolumn{3}{l}{ Fatty acid type and composition ${ }^{\mathrm{a}}(\mathrm{wt} \%)$} & & $\begin{array}{l}\text { Fatty acid ratio, } \\
\text { unsaturated/saturated }\end{array}$ \\
\cline { 2 - 6 } & $\mathrm{C}_{16}: 0$ & $\mathrm{C}_{18}: 0$ & $\mathrm{C}_{20+}$ & $\mathrm{C}_{18}: 1$ & $\mathrm{C}_{18}: 2$ & $\mathrm{C}_{18}: 3$ & \\
\hline Rapeseed & 4 & 6 & 0 & 58 & 22 & 10 & 9 \\
Frying fat & 50 & 7 & 0 & 35 & 8 & 0 & 0.75 \\
Soya oil & 15 & 1 & 0 & 22 & 62 & 0 & 5.25 \\
Peanut oil & 10 & 4 & 5 & 40 & 41 & 0 & 5.8 \\
\hline
\end{tabular}


There are two possible reasons for this behaviour: steric hindering effects on pore diffusion, and the interaction between unsaturated bonds and the catalyst surface. The effective volume of the fatty tail depends strongly on the hydrocarbon chain structure. Saturated chains are more or less straight. Thus, feedstock comprised primarily of such materials should allow easier diffusion inside catalyst, whose structure is rather multi-layer. The "bent" $\mathrm{C}_{18}: 1$ chain (' $\Omega$-9) would be flexible enough for adopting a planar shape. In contrast, the $\mathrm{C}_{18}: 2(' \Omega-6)$ and $\mathrm{C}_{18}: 3 \alpha$-linolenic (' $\Omega$-3) hook-type is more likely to give steric hindering. Unsaturated chains are physically more constrained by hydrogen-catalyst bonds. In the case of peanut oil the presence of $\mathrm{C}_{20+}$ molecules may explain the longer sluggish period.

\subsection{Effects of the Reaction Kinetics on Design and Operation}

Experiments in the micro-plant with different feedstock confirmed the above findings. Two-stage transesterification was performed with full glycerol separation after each step. By keeping the same throughput and alcohol:oil ratio $(9: 1 \mathrm{M})$, an end product with different specifications was obtained. For example, the rapeseed oil gives a conversion of $99.85 \%$ with an ester concentration of $99.5 \%$, exceeding the EN14212 quality specifications. Under identical conditions, soybean oil gives $96 \%$ triglycerides conversion, but with an ester concentration of only $94.3 \%$. The remaining $1.7 \%$ contain mono and di-glycerides that do not conform to the desired norm. One way to solve this problem is by using the variable residence time technology developed by us previously [6].

\section{Conclusions}

Heterogeneous base catalysis offers the most efficient manner for biodiesel manufacturing. Care should be exerted with respect to the dependency of kinetics of the transesterification reaction with feedstock composition. Saturated reaction fatty acid chains give faster reaction rates, while increasing the unsaturation slows down the reaction both by steric and physical bonding effects. Additional hindering might appear due to the presence of larger polyunsaturated molecules.

\section{Experimental Section}

All oil transesterification experiments were carried out under either batch or continuous conditions as follows: Continuous steady-state experiments were performed

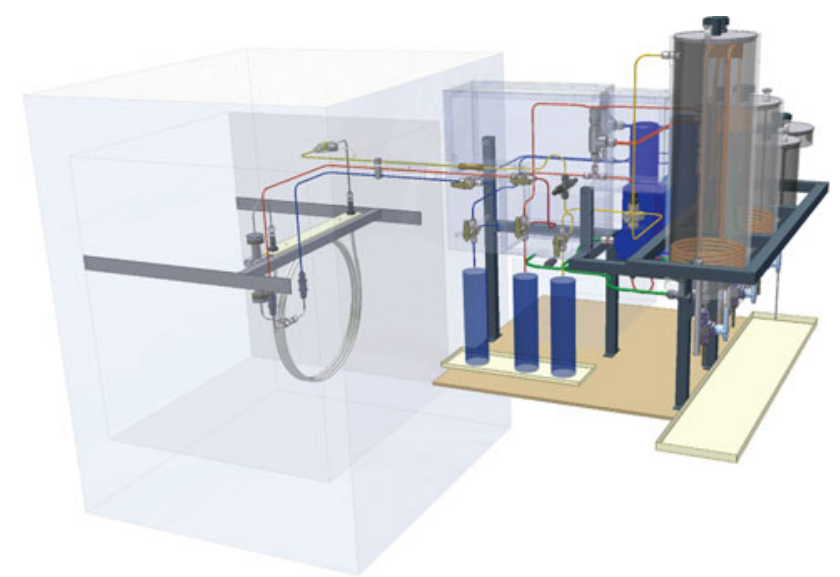

Fig. 5 Schematic of the yellow diesel micro-plant for biodiesel synthesis by heterogeneous catalysis

using our in-house constructed micro-plant (Fig. 5). This features a plug-flow reactor (PFR) coil filled with catalyst grains of $0.8 \mathrm{~mm}$ hosted in an electrical furnace in which temperatures up to $250{ }^{\circ} \mathrm{C}$ can be reached. The feedstock and methanol are fed up in a suitable ratio via a HP pumping and mixing setup. A back-pressure regulator controls the pressure up to $50 \mathrm{bar}$, so that the reaction takes place only in a liquid phase. This micro-plant can produce about $200 \mathrm{~mL} / \mathrm{h}$ bio-diesel of commercial quality (high conversion rates, $>99 \%$, require two consecutive transesterification runs with an intermediate glycerol separation).

Batch experiments were done for testing the catalyst activity and tuning the operating procedure of the continuous plant. A high pressure continuously stirred autoclave of $150 \mathrm{~mL}$ capacity was adapted to deal with solid catalyst in grain form. A typical experiment involves $100 \mathrm{~mL}$ oil, $25 \mathrm{~mL}$ methanol, $10 \mathrm{~mL}$ tetradecane as internal standard and $14 \mathrm{~g}$ catalyst.

For all reactions, products were analysed using an Interscience gas chromatograph equipped with Restek columns, and following EN 14103 protocol for determining glycerides and FAME content.

Open Access This article is distributed under the terms of the Creative Commons Attribution Noncommercial License which permits any noncommercial use, distribution, and reproduction in any medium, provided the original author(s) and source are credited.

\section{References}

1. Kiss AA, Rothenberg G, Dimian AC (2007) Chem Ind (CRC Press, Catal Org React) 115:405

2. Di Serio M, Tesser R, Pengmei L, Santacesaria E (2008) Energy Fuels 22:207

3. Bournay L, Casanave D, Delfort B, Hillion G, Chodorge JA (2005) Catal Today 106:190 
4. Omota F, Dimian AC, Bliek A (2003) Chem Eng Sci 58:3159

5. Kiss AA, Dimian AC, Rothenberg G (2006) Adv Synth Catal 348:75

6. Kiss AA, Omota F, Dimian AC, Rothenberg G (2006) Top Catal 40:141
7. Rothenberg G, Dimian AC (2010) Solid acid catalyst, process for its manufacture, and its use, EP2153893. Yellow Diesel B.V., 17 Feb 2010

8. Om Tapanes NC, Aranda DAG, de Mesquita Carneiro JW, Ceva Antunes OA (2008) Fuel 87:2286 